\title{
Fusidic Acid Resistance Determinants in Methicillin-Resistant Staphylococcus aureus Isolated in Kuwait Hospitals
}

\author{
Halimah A. Boloki Wasmiyah F. Al-Musaileem Wadha AlFouzan \\ Tina Verghese Edet E. Udo \\ Department of Microbiology, Faculty of Medicine, Kuwait University, Kuwait City, Kuwait
}

\section{Highlights of the Study}

- Three genetic determinants, fusA mutation ( $f u s A$ ), $f u s B$, and $f u s C$, were responsible for fusidic acid resistance in methicillin-resistant Staphylococcus aureus isolates from Kuwait hospitals.

- The majority of methicillin-resistant Staphylococcus aureus isolates carried fusC.

- Methicillin-resistant Staphylococcus aureus strains belonged to diverse genetic backgrounds.

- The high prevalence of fusidic acid-resistant methicillin-resistant Staphylococcus aureus calls for a review of fusidic acid usage to preserve the therapeutic benefit of the antibiotic.

\section{Keywords}

Fusidic acid resistance $\cdot$ Methicillin-resistant Staphylococcus aureus $\cdot$ Spa typing $\cdot$ DNA microarray

\begin{abstract}
Objective: The aim of this study was to investigate the genetic determinants of fusidic acid (FA) resistance in MRSA isolated from patients in Kuwait hospitals. Methods: The minimum inhibitory concentration (MIC) of FA was tested with E-test strips. Genetic determinants of FA were determined by PCR and DNA microarray. Staphylococcal protein A gene (spa) typing and DNA microarray analysis were used to study their genetic backgrounds. Results: The FA MIC ranged from $2 \mathrm{mg} / \mathrm{L}$ to $>256 \mathrm{mg} / \mathrm{L}$. Of the 97 isolates, 79 (81.4\%) harbored fusC, 14 isolates harbored fusA mutations
\end{abstract}

(fus $A)$, and 4 isolates harbored fusB. Isolates with fus $A$ mutations expressed high FA MIC (MIC $>256 \mathrm{mg} / \mathrm{L}$ ), whereas those with fusC and fusB expressed low FA MIC (MIC 2-16 mg/L). The isolates belonged to 23 spa types and 12 clonal complexes (CCs). The major spa types were t688 $(n=25), \mathrm{t} 311$ ( $n$ $=14), \mathrm{t} 860(n=8)$, and $\mathrm{t} 127(n=6)$ which constituted $54.6 \%$ of the isolates. The 12 CCs were CC1, CC5, CC8, CC15, CC22, CC80, CC88, and CC97 with CC5 (45.6\%) and CC97 (13.2\%) as the dominant CCs. Conclusions: The MRSA isolates belonged to diverse genetic backgrounds with the majority carrying the fusC resistance determinants. The high prevalence of FA resistance belonging to diverse genetic backgrounds warrants a review of FA usage in the country to preserve its therapeutic benefits.

(C) 2021 The Author(s). Published by S. Karger AG, Basel karger@karger.com www.karger.com/mpp

Karger $\stackrel{\text { ' }}{5}$

GOPEN ACCESS
C 2021 The Author(s).

Published by S. Karger AG, Basel

This is an Open Access article licensed under the Creative Commons Attribution-NonCommercial-4.0 International License (CC BY-NC) (http://www.karger.com/Services/OpenAccessLicense), applicable to the online version of the article only. Usage and distribution for commercial purposes requires written permission.
Correspondence to:

Edet E. Udo, udo.ekpenyong@ku.edu.kw 


\section{Introduction}

Staphylococcus aureus is an opportunistic bacterial pathogen that is capable of causing a wide range of infections, including superficial skin and soft tissue infections (SSTIs), pneumonia, bloodstream infections, and bone and joint, respiratory tract, and gastrointestinal tract as well as urinary tract infections [1-3]. Uncomplicated SSTIs are often treated with topical antibiotics such as fusidic acid (FA) and mupirocin [1,2]. Topical antibiotics offer effective treatment options for SSTIs because the antibiotics are localized, well tolerated, easy to administer, and easily available over the counter $[1,2]$. FA is a steroid antibiotic that was introduced for clinical use in the $1960 \mathrm{~s}$ and has been used clinically for the systemic and topical treatment of staphylococcal infections $[1,2]$. FA kills bacteria by inhibiting protein synthesis by binding to the ribosomal translocase elongation factor G (EF-G) [4]. Since EF-G is involved in the translocation part of translation, it forms a complex with guanosine triphosphatase catalyzing the movement of tRNA and mRNA through the ribosome. FA binds to EF-G and blocks the elongation of the polypeptide chain and EF-G-guanosine dinucleotide ribosomal complex release $[4,5]$.

Resistance to FA appeared following the extensive and widespread use of the antibiotic and has been reported in clinical isolates of $S$. aureus obtained in different countries [6-11]. Three common genetic determinants (fus $A$ [mutation in $f u s A$ ], fus $B$, and $f u s C$ ) mediate FA resistance in $S$. aureus. These determinants vary in their genetic locations. fus $A$ confers chromosomal resistance $[5,11,12]$, while $f u s B$ and $f u s C$ are cytoplasmic and are located on plasmids or transposon-like elements $[5,6,12-15]$ although chromosomal fusB was also reported in the European FA-resistant $S$. aureus impetigo clone $[7,8]$.

The prevalence of resistance to FA in $S$. aureus varies in different countries $[8-11,13]$. In Kuwait, the prevalence of FA resistance has remained consistently high among clinical MRSA isolates [16-18]. A study of antibiotic resistance in S. aureus obtained in 21 laboratories located in 19 countries conducted in 1996 discovered that the prevalence of FA resistance in $S$. aureus obtained in Kuwait was $20 \%$ which was higher than the global average of $5 \%$ at the time [19]. Since then, the prevalence of FA resistance has continued to increase among MRSA isolates in Kuwait $[10,17,18]$ and in other countries $[8,9$, $11,13]$ making FA resistance a major resistance problem in MRSA isolated globally.

The recent increase in FA resistance in $S$. aureus in many countries has been attributed to the emergence of novel genetic elements comprising fus $C$ inserted into the staphylococcal cassette chromosome mec (SCCmec) region of MRSA strains. The SCCmec region harbors the methicillin resistance gene ( $m e c A)$, and the integration of fusC into the SCCmec region is suggested to enhance its rapid spread in MRSA belonging to different genetic backgrounds $[11,13,15,18]$.

Although FA resistance in MRSA obtained in Kuwait hospitals has been well documented [10, 16-18], data on the genetic determinants of FA resistance in Kuwait MRSA isolates are limited [18]. Understanding the mechanisms of FA resistance will be helpful for effective and appropriate use of the antibiotic. The aim of this study was to investigate the genetic determinants of FA resistance in MRSA strains collected from patients in Kuwait hospitals.

\section{Materials and Methods}

\section{MRSA Isolates}

The MRSA isolates were obtained as part of routine diagnostic microbiology investigations in 12 public hospitals using standard bacteriological protocols. The isolates were later submitted to the MRSA Reference Laboratory situated at the Department of Microbiology, Faculty of Medicine, Kuwait University, Kuwait, for molecular typing. Prior antibiotic susceptibility testing of 1044 MRSA isolates obtained between January 1 and June 30, 2018 , revealed that $97(9.2 \%)$ isolates were resistant to FA. The FA-resistant isolates were preserved in $15 \%$ glycerol $(\mathrm{v} / \mathrm{v}$ in brain heart infusion broth (Oxoid, Basingstoke, Leicester, UK) at $-80^{\circ} \mathrm{C}$ and were investigated further in this study. The isolates were recovered by growth in brain heart infusion broth at $35^{\circ} \mathrm{C}$ for $24 \mathrm{~h}$ followed by 2 further subcultures on brain heart infusion agar [18].

\section{Antibiotic Susceptibility Testing}

Antibiotic susceptibilities of the FA-resistant isolates were confirmed by the disk diffusion method on Mueller-Hinton Agar (Oxoid, Basingstoke, UK) and incubated for $24 \mathrm{~h}$ at $35^{\circ} \mathrm{C}$. The following antibiotic discs were tested: cefoxitin $(30 \mu \mathrm{g})$, penicillin $(10$ $\mathrm{U})$, trimethoprim $(5 \mu \mathrm{g})$, tetracycline $(30 \mu \mathrm{g})$, kanamycin $(30 \mu \mathrm{g})$, gentamicin $(10 \mu \mathrm{g})$, erythromycin $(15 \mu \mathrm{g}), \mathrm{FA}(10 \mu \mathrm{g})$, chloramphenicol $(30 \mu \mathrm{g})$, rifampicin $(5 \mu \mathrm{g})$, linezolid $(30 \mu \mathrm{g})$, and mupirocin $(200 \mu \mathrm{g})$. Minimum inhibitory concentration (MIC) for cefoxitin, mupirocin, vancomycin, teicoplanin, and FA was determined with E-test strips (AB BioMerieux, Marcy l' Etoile, France) according to the manufacturer's instructions and interpreted according to the European Committee on Antimicrobial susceptibility testing [20]. S. aureus strains ATCC 25923 and ATCC 29213 were used as quality control strains for disk diffusion and MIC determinations, respectively. Methicillin resistance was confirmed by detecting PBP2A in culture supernatants of isolates using a rapid latex agglutination kit (Denka-Seiken, Tokyo, Japan) following the manufacturer's instructions [17]. 


\section{Molecular Typing of MRSA Isolates}

Isolation of bacterial DNA for amplification studies was performed as described previously [18]. Three to 5 identical colonies of an overnight culture were picked using a sterile loop and suspended in a microfuge tube containing $50 \mu \mathrm{L}$ of lysostaphin (150 $\mu \mathrm{g} / \mathrm{mL})$ and $10 \mu \mathrm{L}$ of RNase $(10 \mu \mathrm{g} / \mathrm{mL})$ solution. The tube was incubated at $37^{\circ} \mathrm{C}$ in the heating block (ThermoMixer, Eppendorf, Hamburg, Germany) for $20 \mathrm{~min}$. To each sample, $50 \mu \mathrm{L}$ of proteinase $\mathrm{K}(20 \mathrm{mg} / \mathrm{mL})$ and $150 \mu \mathrm{L}$ of Tris buffer $(0.1 \mathrm{M})$ were added and mixed by pipetting. The tube was then incubated at $60^{\circ} \mathrm{C}$ in the water bath (VWR Scientific Co., Shellware Lab, Radnor, PA, USA) for $10 \mathrm{~min}$. The tube was transferred to a heating block at $95^{\circ} \mathrm{C}$ for 10 min to inactivate proteinase $\mathrm{K}$ activity. Finally, the tube was centrifuged, and the extracted DNA was stored at $4^{\circ} \mathrm{C}$ till used for PCR.

\section{Spa Typing}

Spa typing was performed as described previously by Harmsen et al. [21]. The PCR protocol consisted of an initial denaturation at $94^{\circ} \mathrm{C}$ for $4 \mathrm{~min}$, followed by 25 cycles of denaturation at $94^{\circ} \mathrm{C}$ for $1 \mathrm{~min}$, annealing at $56^{\circ} \mathrm{C}$ for $1 \mathrm{~min}$, and extension for $3 \mathrm{~min}$ at $72^{\circ} \mathrm{C}$, and a final cycle with a single extension for $5 \mathrm{~min}$ at $72^{\circ} \mathrm{C}$. Five microliters of the PCR product was analyzed by $1.5 \%$ agarose gel electrophoresis to confirm amplification. The amplified PCR product was purified using the MicroElute Cycle-Pure Spin kit (Omega Bio-tek, Inc., Norcross, GA, USA), and the purified DNA was then used for sequencing PCR. The sequencing PCR product was then purified using the Ultra-Sep Dye Terminator Removal kit (Omega Bio-tek, Inc., Norcross, GA, USA). The purified DNA was sequenced in an automated 3130X1 genetic analyzer (Applied Biosystem, Waltham, MA, USA). The sequenced spa gene was analyzed using Ridom Staph Type software available on Ridom SpaServer at https//spaserver.ridom.de.

\section{DNA Microarray}

All 97 FA-resistant isolates were investigated by DNA microarray using the $S$. aureus genotyping kit (Alere, Technology, Jena, Germany) for the molecular typing of S. aureus isolates. The method allows for the detection of genes for antibiotic resistance and virulence factors and the assignment of the isolates to clonal complexes (CCs). SCCmec types of the isolates were derived from DNA microarray analysis. The procedures were performed according to the protocol published previously $[18,22]$.

\section{Amplification of FA Resistance Genes}

The presence of fusA (mutation in fusA), fusB, and fusC was investigated by PCR using primers and methods published previously $[5,23]$. fusA mutation was amplified with primers FusA-F $\left(5^{\prime}\right.$-CTC GTA AYA TCG GTA TCA TG- $\left.3^{\prime}\right)$ and FusA-R $\left(5^{\prime}\right.$-GCA TAG TGA TCG AAG TAC- $3^{\prime}$ ), fus $C$ was amplified with primers FusC-F ( $5^{\prime}$-TTA AAG AAA AAG ATA TTG ATA TCT CGG-3') and FusC-R ( $5^{\prime}$-TTT ACA GAA TCC TTT TAC TTT ATT TGG$\left.3^{\prime}\right)$, and for $f u s B$, the primers FarlA $\left(5^{\prime}\right.$-ACT TGT CCG TGT GCT AAC- $\left.3^{\prime}\right)$ and Far1B ( $5^{\prime}$-AGT TCG GGA GGT GAT GATG-3') were used. The cycling conditions consisted of an initial denaturation step $\left(94^{\circ} \mathrm{C}\right.$ for $\left.3 \mathrm{~min}\right)$, followed by 25 cycles of $94^{\circ} \mathrm{C}(30 \mathrm{~s})$, $57^{\circ} \mathrm{C}(30 \mathrm{~s})$, and $72^{\circ} \mathrm{C}(45 \mathrm{~s})$ [5]. The PCR products were subjected to agarose gel electrophoresis in $1.5 \%(\mathrm{w} / \mathrm{v})$ agarose gels containing ethidium bromide $(0.5 \mathrm{mg} / \mathrm{L})$ and visualized under ultraviolet light. The expected amplicon sizes in base pairs were fusA, 2,002
Table 1. Distribution of FA resistance determinants in MRSA isolates

\begin{tabular}{rrrrrr}
\hline & \multirow{2}{*}{$\begin{array}{l}\text { MIC, } \\
\mathrm{mg} / \mathrm{L}\end{array}$} & \multicolumn{5}{c}{ FA resistance determinants } \\
\cline { 3 - 6 } & & fusA & fusB & fusC & total \\
\hline 1 & 2 & - & - & & 1 \\
2 & 4 & - & 4 & 76 & 80 \\
3 & 12 & - & - & 1 & 1 \\
4 & 16 & - & - & 1 & 1 \\
5 & $\geq 256$ & 14 & - & - & 14 \\
\cline { 2 - 6 } & Total & 14 & 4 & 79 & 97 \\
\hline
\end{tabular}

MRSA, methicillin-resistant Staphylococcus aureus; FA, fusidic acid; MIC, minimum inhibitory concentration.

bp; fusB, $949 \mathrm{bp}$; and fusC, $332 \mathrm{bp}$ [23]. Detection of mutations in fusA was performed by the rapid PCR amplification method for the detection of the L461K mutation in fus $A$ as described previously by Castanheira et al. [23]. The amplification was performed using 4 primer sets [23]. PCR was performed in $20 \mu \mathrm{L}$ using Master Mix (5X HOT FIRE Pol Blend, with $7.5 \mathrm{mM} \mathrm{MgCl}_{2}$; Solis Biodyne, Tartu, Estonia), $0.1-0.4 \mu \mathrm{M}$ of primers, and $2 \mu \mathrm{L}$ of the template. Cycling conditions were cell denaturation of $15 \mathrm{~min}$ at $95^{\circ} \mathrm{C}$, followed by 35 cycles of $15 \mathrm{~s}$ at $94^{\circ} \mathrm{C}, 30 \mathrm{~s}$ at $50^{\circ} \mathrm{C}$, and $45 \mathrm{~s}$ at $72^{\circ} \mathrm{C}$ for denaturing, annealing, and extension, respectively, and final extension at $72^{\circ} \mathrm{C}$ for $5 \mathrm{~min}$. PCR products were visualized after $2 \%$ agarose gel electrophoresis. Two bands were detected in strains positive for L461K with 1 band of $415 \mathrm{bp}$ and another with $145 \mathrm{bp}$. L461K-negative strains had 2 bands of $415 \mathrm{bp}$ and $308 \mathrm{bp}$ [23].

\section{Results}

The 97 FA-resistant MRSA isolates were obtained from wound swabs $(n=23)$, blood $(n=17)$, nasal swabs $(n=12)$, endotracheal aspirate $(n=6)$, urine $(n=5)$, groin $(n=5)$, axilla $(n=4)$, high vaginal swab $(n=3)$, umbilical cord swab $(n=3)$, throat swab $(n=3)$, ear swab $(n=3)$, eye swab (2), skin $(n=2)$, tissue $(n=2)$, sputum $(n=2)$, exit site $(n=1)$, fluid $(n=1)$, and unspecified sources $(n=2)$.

\section{Antibiotic Resistance of FA-Resistant MRSA Isolates}

The distribution of FA MIC levels is summarized in Table 1 . The FA MICs ranged from $2 \mathrm{mg} / \mathrm{L}$ to $>256 \mathrm{mg} / \mathrm{L}$. Most of the isolates expressed low FA MICs (MIC 2-16 mg/L). Fourteen isolates expressed high FA MIC levels (MIC > 256 $\mathrm{mg} / \mathrm{L}$ ). In addition to FA resistance, the MRSA isolates were resistant to the nonbeta-lactam antibiotics shown in Table 2. Most of the isolates were resistant to trimethoprim $(n=47)$ and tetracycline $(n=43)$. Of the 27 clindamycin-resistant 


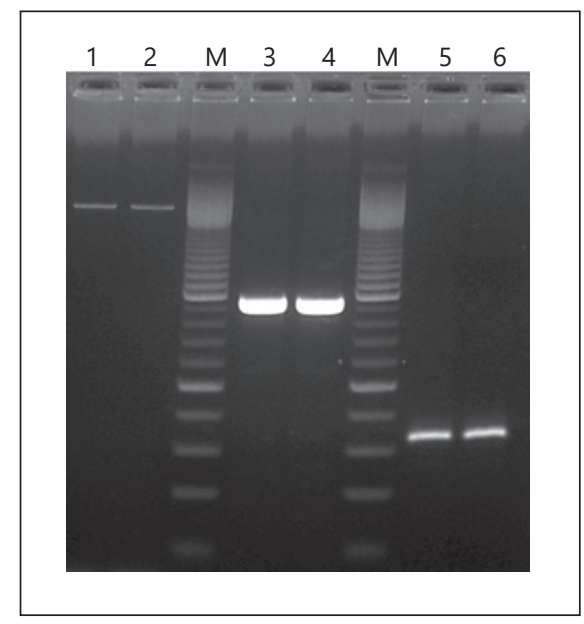

Fig. 1. Agarose gel electrophoresis of amplified FA resistance determinants. Lanes 1 and 2 fusA (size 2,002 bp); lane M, molecular size marker - 100 bp ladders; lanes 3 and 4 fus $B$ (size 949 bp); lanes 5 and 6 fus C (size 332 bp). FA, fusidic acid.

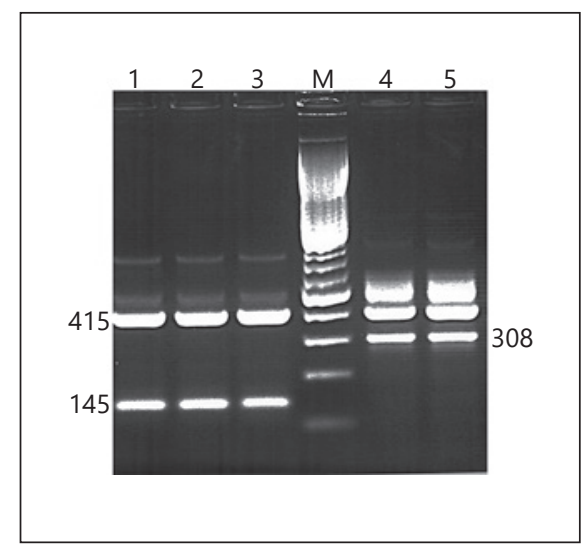

Fig. 2. Agarose gel electrophoresis showing the L416K mutation. Lanes 1-3 fusA mutation (L416K); lane M molecular size marker (100 bp); lanes 4 and 5 no L416K mutation.

isolates, 20 expressed inducible resistance, while 7 expressed constitutive resistance. Twenty-five isolates were resistant to chloramphenicol. All isolates were susceptible to vancomycin (MIC $\leq 2 \mathrm{mg} / \mathrm{L}$ ), teicoplanin ( $\mathrm{MIC} \leq 2 \mathrm{mg} / \mathrm{L}$ ), linezolid, rifampicin, and mupirocin.

\section{Prevalence of FA Resistance Determinants}

The distribution of the FA resistance determinants is summarized in Table 1. Figure 1 shows representative amplified products of the FA resistance determinants. The FA resistance determinants, fusA (fusA mutation),
Table 2. Prevalence of antibiotic resistance in FA-resistant MRSA isolates

\begin{tabular}{ll}
\hline Antibiotics & MRSA (\%) \\
\hline Trimethoprim & $47(48.9)$ \\
Tetracycline & $43(44.8)$ \\
Kanamycin & $32(33.3)$ \\
Gentamicin & $28(29.1)$ \\
Erythromycin & $27(28.1)$ \\
Clindamycin & $27(28.1)$ \\
Chloramphenicol & $25(25.7)$ \\
\hline
\end{tabular}
acid.

MRSA, methicillin-resistant Staphylococcus aureus; FA, fusidic

$f u s B$, and $f u s C$, were investigated by PCR amplification. In addition, results for $f u s B$ and $f u s C$ were extracted from DNA microarray analysis of the isolates as the DNA Microarray platform used in this study also contains probes for $f u s B$ and $f u s C$. There was complete agreement in the results obtained by PCR and DNA microarray analysis for fus $C$ and fus $B$. Table 1 contains the results obtained by both methods. Most of the isolates $(n=79 ; 81.4 \%)$ were positive for fus $C, 14$ isolates harbored fus $A$, and 4 isolates (4.1\%) harbored fusB. One isolate was positive for both fusA and fus $C$. The association of the FA resistance determinants with FA MIC revealed that fusA was associated with high MIC values (MIC $>256 \mathrm{mg} / \mathrm{L}$ ) while $f u s B$ and fus $C$ were associated with lower FA MIC values.

\section{Detection of fusA Mutations}

All 97 FA-resistant isolates were investigated for fus $A$ mutations. The result is presented in Figure 2. The L461K mutation was detected only in the 14 isolates with highlevel FA resistance (MIC >256 mg/L).

\section{Genetic Backgrounds of FA-Resistant MRSA Isolates}

The genetic backgrounds of the 97 FA-resistant isolates are presented in Table 3. The isolates were classified into 5 SCCmec types: SCCmec III, SCCmec IV, SCCmec $\mathrm{V}$, SCCmec VI, and SCCmec V/VT; 23 spa types; $12 \mathrm{CCs}$; and 26 genotypes.

The dominant spa types were t688 $(n=25), \mathrm{t} 311(n=$ $14), \mathrm{t} 860(n=8)$, and $\mathrm{t} 127(n=6)$ which constituted $54.6 \%$ of the spa types. Spa types, t084, t362, and t044 were detected in 4 isolates each while t3841, t535, and t 267 were each detected in 3 isolates. The remaining spa types were detected in 2 or single isolates. The spa types of 4 isolates were not determined. 
Table 3. Genetic determinants of FA resistance in MRSA isolates

\begin{tabular}{|c|c|c|c|c|c|c|}
\hline \multirow[t]{2}{*}{ Clonal complex } & \multirow[t]{2}{*}{ Strain description } & \multirow[t]{2}{*}{ Spa types } & \multicolumn{4}{|c|}{ FA resistance determinants } \\
\hline & & & fusA & fusB & fusC & total \\
\hline \multirow[t]{2}{*}{ CC1 } & CC1-MRSA-V + SCCfus $\left[\mathrm{PVL}^{+}\right]$ & $\mathrm{t} 127$ & & & 5 & 5 \\
\hline & CC1-MRSA-IV + SCCfus & $\mathrm{t} 127$ & & & 1 & 1 \\
\hline \multirow[t]{7}{*}{ CC5 } & CC5-MRSA-VI + SCCfus & t688 & 1 & & 24 & 25 \\
\hline & CC5-MRSA-V + SCCfus & t3235 & & & 1 & 1 \\
\hline & CC5-MRSA-VI + SCCfus & $\mathrm{t} 2235$ & & & 2 & 2 \\
\hline & CC5-MRSA-V + SCCfus & $\mathrm{t} 311$ & & & 13 & 13 \\
\hline & CC5-MRSA-V-[sed/j] & t954 & & & 1 & 1 \\
\hline & CC5-MRSA-V + SCCfus & $\mathrm{t} 442$ & & & 1 & 1 \\
\hline & CC5-MRSA-VI + SCCfus & $\mathrm{t} 535$ & & & 3 & 3 \\
\hline \multirow[t]{3}{*}{ CC8 } & ST239-MRSA-III + SCCmer & $\mathrm{t} 860$ & 8 & & & 8 \\
\hline & ST239-MRSA-III + SCCmer & t945 & 2 & & & 2 \\
\hline & CC8-MRSA-IV [PVL $\left.{ }^{+}\right]$ & t008 & & & 1 & 1 \\
\hline CC15 & CC15-MRSA-V + SCCfus & $\mathrm{t} 084$ & & & 4 & 4 \\
\hline \multirow[t]{2}{*}{ CC22 } & CC22-MRSA-IV [fnbB; sec/I & $\mathrm{t} 311$ & 1 & & & 1 \\
\hline & C22-MRSA-IV [tst1] & $\mathrm{t} 1612$ & & & 1 & 1 \\
\hline CC30 & CC30-MRSA-VI + fus $\left[\mathrm{PVL}^{+}\right]$ & t018 & & & 2 & 2 \\
\hline CC45 & CC45-MRSA-VI + fus & $\mathrm{t} 362$ & & & 4 & 4 \\
\hline CC80 & CC80-MRSA-IV $\left[\mathrm{PVL}^{+}\right]$ & t044 & & 4 & & 4 \\
\hline \multirow[t]{2}{*}{ CC88 } & CC88-MRSA-IV & t690 & & & 1 & 1 \\
\hline & CC88-MRSA-IV[etA $\left.{ }^{+}\right]$ & t786 & 2 & & & 2 \\
\hline \multirow[t]{4}{*}{ CC97 } & CC97-MRSA-V[fusC] & t2297 & & & 2 & 2 \\
\hline & CC97-MRSA-V[fusC] & t359 & & & 2 & 2 \\
\hline & CC97-MRSA-V[fusC] & $\mathrm{t} 267$ & & & 3 & 3 \\
\hline & CC97-MRSA-V[fusC] & nd & & & 4 & 4 \\
\hline CC152 & CC152-MRSA-[V/VT $\left.+\mathrm{PVL}^{+}\right)$ & $\mathrm{t} 353$ & & & 1 & 1 \\
\hline CC361 & CC361-MRSA-V/VT + fus & t3841 & & & 3 & 3 \\
\hline Total & & & 14 & 4 & 79 & 97 \\
\hline
\end{tabular}

MRSA, methicillin-resistant Staphylococcus aureus; FA, fusidic acid; nd, not determined.

Most of the isolates belonged to SCCmec V $(n=36)$ or SCCmec VI $(n=36)$ while 11 and 10 isolates belonged to SCCmec IV and SCCmec III, respectively. CC5 was the dominant CC. It comprised 44 isolates grouped into 7 genotypes. The other major CCs were CC8 (ST239) (11.3\%), CC97 (11.3\%), CC1 (6.1\%), CC15 (5.1\%), CC45 (4.1\%), CC80 (4.1\%), and CC88 (4.1\%). The remaining CCs were less common (Table 3 ).

The common genotypes were CC5-MRSA-VI + SCCfus-t688 $(n=25)$, CC5-MRSA-V + SCCfus-t311 $(n=$ 13), ST239-MRSA-III-t860 ( $n=8)$, CC1-MRSA-V + SC-
Cfus [PVL+] $(n=5)$, CC15-MRSA-V + SCCfus $(n=4)$, CC45-MRSA-VI + fus $(n=4)$, and CC80-MRSA-IV $[\mathrm{PVL}+](n=4)$. The other genotypes were detected in $1-3$ isolates.

The distribution of the FA resistance determinants by genotypes showed that $f u s C$ was the most widely distributed FA resistance determinant. fus $C$ was detected in all CCs except CC8 (ST239), CC80, and CC88. fusB was only detected in CC80 isolates while fusA mutation was found in CC5 $(N=1), \operatorname{CC} 22(N=1), \operatorname{CC} 88(N=2)$, and CC8 (ST239) $(N=10)$. 


\section{Discussion}

This study investigated the genetic mechanisms of FA resistance in clinical MRSA isolates obtained in Kuwait hospitals. The results revealed the presence of the $3 \mathrm{com}$ mon FA resistance determinants, fusA (fusA mutation), $f u s B$, and $f u s C$, that have been associated with FA resistance in S. aureus [5-9]. The study showed that fus $C$, detected in $81.4 \%$ of the isolates, was the dominant FA resistance determinant. This observation is consistent with results from other studies where $f u s C$ was more prevalent among FA-resistant MRSA isolates in Taiwan [15] and in MRSA obtained from patients with atopic dermatitis in Denmark [24].

The fusA mutation, detected in $14.4 \%$ of the isolates, was the second common FA resistance determinant in this study. The isolates with mutations in fusA expressed high-level FA resistance (MIC $>256 \mathrm{mg} / \mathrm{L}$ ) and harbored the L416K mutation. Although different mutations have been associated with $f u s A$, the $\mathrm{L} 461 \mathrm{~K}$ mutation is more common in $S$. aureus $[23,25]$. Therefore, the high-level FA-resistant isolates were investigated for the presence of the L416K mutations, and all 14 high-level FA-resistant isolates carried the $\mathrm{L} 416 \mathrm{~K}$ mutation. The association of high-level FA resistance with fusA mutations observed in this study is consistent with previous studies from Europe $[5,24]$ and Taiwan [25] that had associated fusA with high-level FA resistance (MIC $\geq 64 \mathrm{mg} / \mathrm{L}$ ) and $f u s B$ or fus $C$ with low-level resistance (MIC $<32 \mathrm{mg} / \mathrm{L}$ ).

The fus $B$ determinant was detected in the least number $(4.1 \%)$ of isolates in this study. In contrast, fusB was the most prevalent FA resistance determinant in MRSA isolated in Jordan [26], Wenzhou, China [27], and in some European countries due to an epidemic strain associated with outbreaks of impetigo bullosa infections and clonal expansion of the European CA-MRSA clone belonging to ST $80[7,8,13,24]$. These reports highlight differences in the geographical distribution of FA resistance determinants in MRSA isolates.

One isolate with high-level FA resistance harbored both fus $A$ and fus $C$ determinants suggesting that the high MIC expressed by fusA was probably masking the lower MIC associated with fusC. The simultaneous carriage of double FA resistance determinants in individual strains is rare. Previous reports include the simultaneous carriage of $f u s B+f u s C$ in one of $25 \mathrm{MRSA}$ isolates and in 2 of 34 FA-resistant MRSA in separate Taiwanese hospitals [28], the presence of fusB + fus $C$ in one of 26 FA-resistant MRSA in Egypt [29], and the presence of fusB + fusA (2 isolates) and fusC + fusA ( 1 isolate) in MRSA isolates in Denmark [7]. The simultaneous

Fusidic Acid Resistance Determinants in MRSA Isolates carriage of fus $A+f u s C$ in this study adds to the growing list of this phenomenon. Similar to the observation in this study, in vitro studies by O'Neill and Chopra [6] revealed that when a plasmid-mediated $f u s B$ was introduced into a strain carrying chromosomally mutated fusA, it retained the MIC values of the determinant exhibiting the highest MIC values (fusA). A similar observation was reported in clinical MRSA isolates carrying fus $C+f u s A$ or $f u s B+f u s A$ where the FA MIC corresponded to the MIC expressed by fus $A$ [5]. Furthermore, a report of a study from Egypt revealed that when an FAresistant MRSA which carried both fusB and fusC and expressed high-level resistance (MIC 1,024 mg/L) was cured of the plasmid-mediated fusB, the loss of the plasmid resulted in the reduction of FA MIC values by 16-64-folds suggesting that the FA resistance in this isolate was additive [29]. These results highlight the complexities of FA resistance determinants in MRSA isolates [5, 6].

This study revealed that the FA-resistant MRSA isolates belonged to diverse genetic backgrounds consisting of 23 spa types, $12 \mathrm{CCs}$, and 16 genotypes with CC5 (48.4\%) as the dominant CC followed by CC97 (11.3\%) and CC8 (11.3\%). The dominant genotypes were CC5MRSA-VI + SCCfus-t688 (25.7\%), CC5-MRSA-V + SCCfus-t311 (13.4\%), and CC8 (ST239-MRSA-III-t860) (8.2\%). Furthermore, while fusC was widely distributed among the different genetic backgrounds, fusB was limited to CC80, and fusA that was associated with high-level FA resistance (MIC $>256 \mathrm{mg} / \mathrm{L}$ ) was restricted to ST239-MRSA-III, CC5, CC22, and CC88 isolates. Prior to this study, Boswihi et al. [18] reported the presence of fusC in $87.2 \%$ of novel variants of MRSA that belonged to diverse genetic backgrounds in Kuwait. Hence, the current detection of fus $C$ in 10 of the 12 CCs confirms an ongoing expansion of $f u s C$ among FA-resistant MRSA in Kuwait hospitals. Similarly, Ellington et al. [13] found fusC in CA-MRSA belonging to different genetic backgrounds including ST1, ST5, ST8, ST45, and ST149, while FA-resistant MRSA carrying fusC in Malaysia belonged to multiple genetic backgrounds [15], and FA-resistant MRSA obtained from children in Greece belonged to ST80, ST30, and ST22 [30]. These results highlight the growing contribution of $f u s C$ to the increasing prevalence of FA resistance in $S$. aureus.

\section{Conclusion}

The study has revealed a high prevalence of $f u s C$ in FAresistant MRSA belonging to diverse genetic backgrounds. This study has increased our understanding of 
the genetic determinants of FA resistance in MRSA isolated in Kuwait hospitals. The increasing prevalence of FA resistance and the evolution of the combination of fus $C$ with SCCmec genetic elements observed in this and other studies warrant a review of topical FA use in the country so as not to completely lose its efficacy against $S$. aureus.

\section{Acknowledgments}

We are grateful to the staff of the MRSA Reference Laboratory for technical support.

\section{Statement of Ethics}

The strains used in this study were obtained as part of routine diagnostic services. Therefore, no ethical approval was required.

\section{Conflict of Interest Statement}

The authors have no conflicts of interest.

\section{Funding Sources}

There was no external funding for this study.

\section{Author Contributions}

E.E.U. and W.A. initiated the study. H.A.B., F.A.M., and T.V. performed the experiments. All authors contributed to data analysis, drafting, and revising the manuscript and approved the final version for publication.

\section{Data Availability Statement}

All relevant data are available within the text.

\section{References}

1 Pangilinan R, Tice A, Tillotson G. Topical antibiotic treatment for uncomplicated skin and skin structure infections: review of the literature. Expert Rev Anti Infect Ther. 2009;7: 957-65.

2 Williamson DA, Carter GP, Howden BP. Current and emerging topical antibacterial and antiseptic: agents, action, and resistance patterns. Clin Microbiol Rev. 2017;30:82760.

3 Gajdács M, Ábrók M, Lázár A, Burián K. Increasing relevance of gram-positive cocci in urinary tract infections: a 10-year analysis of their prevalence and resistance trends. Sci Rep. 2020 Oct 19;10(1):17658.

4 Bodley JW, Zieve FJ, Lin L, Zieve ST. Formation of the ribosome-G factor-GDP complex in the presence of fusidic acid. Biochem Biophys Res Commun. 1969;37:437-43.

5 McLaws FB, Larsen AR, Skov RL, Chopra I, O'Neill AJ. Distribution of fusidic acid resistance determinants in methicillin-resistant Staphylococcus aureus. Antimicrob Agents Chemother. 2011;55:1173-6.

6 O'Neill AJ, Chopra I. Molecular basis of fusBmediated resistance to fusidic acid in Staphylococcus aureus. Mol Microbiol. 2006;59: 664-76.

7 O’Neill AJ, Larsen, Henriksen AS, Chopra I. A fusidic acid: resistant epidemic strain of Staphylococcus aureus carries fusB determinant, whereas fusA mutations are prevalent in other resistant isolates. Antimcrob Agents Chemother. 2004;48:3594-7.

8 Rijnders MI, Wolffs PF, Hopstaken RM, den Heyer M, Bruggeman CA, Stobberingh EE. Spread of the epidemic European fusidic acidresistant impetigo clone (EEFIC) in general practice patients in the south of The Netherlands. J Antimicrob Chemother. 2012;67: 1176-80.

9 Williamson DA, Monecke S, Heffernan H, Ritchie SR, Roberts SA, Upton A, et al. High usage of topical fusidic acid and rapid clonal expansion of fusidic acid-resistant Staphylococcus aureus: a cautionary tale. Clin Infect Dis. 2014;59:1451-4.

10 Udo EE, Boswihi SS. Antibiotic resistance trends in methicillin-resistant Staphylococcus aureus isolated in Kuwait hospitals: 20112015. Med Princ Pract. 2017;26:485-90.

11 Baines SL, Howden BP, Heffernan H, Stinear TP, Carter GP, Seemann T, et al. Rapid emergence and evolution of staphylococcus aureus clones harboring fusC-containing staphylococcal cassette chromosome elements. Antimicrob Agents Chemother. 2016;60:2359-65.

12 Gajdacs M. The continuing threat of methicillin-resistant Staphylococcus aureus. Antibiotics. 2019;8:52.

13 Ellington MJ, Reuter S, Harris SR, Holden MT, Cartwright EJ, Greaves D, et al. Emergent and evolving antimicrobial resistance cassettes in community-associated fusidic acid and meticillin-resistant Staphylococcus aureus. Int J Antimicrob Agents. 2018;45: 477-84.

14 Lim KT, Teh CS, Yusof MY, Thong KL. Mutations in rpoB and fusA cause resistance to rifampicin and fusidic acid in methicillin-resistant Staphylococcus aureus strains from a tertiary hospital in Malaysia. Trans R Soc Trop Med Hyg. 2014;108:112-8.

15 Lin YT, Tsai JC, Chen HJ, Hung WC, Hsueh PR, Teng LJ. A novel staphylococcal cassette chromosomal element, SCCfusC, carrying
fusC and speG in fusidic acid-resistant methicillin-resistant Staphylococcus aureus. Antimicrob Agents Chemother. 2014;58:1224-7.

16 Udo EE, Jacob LE. Characteristics of methicillin-resistant Staphylococcus aureus from $\mathrm{Ku}$ wait hospitals with high-level fusidic acid resistance. J Med. Microbiol. 2000;49:419-26.

17 Udo EE, Al-Sweih N, Dhar R, Dimitrov TS, Mokaddas EM, Johny M, et al. Surveillance of antibacterial resistance in Staphylococcus aureus isolated in Kuwaiti hospitals. Med Princ Pract. 2008;17:71-5.

18 Boswihi SS, Udo EE, Monecke S, Mathew B, Noronha B, Verghese T, et al. Emerging variants of methicillin-resistant Staphylococcus aureus genotypes in Kuwait hospitals. PLoS One. 2018;13(4):e0195933.

19 Zinn CS, Westh H, Rosdahl VT; The SARISA Study Group. An international multicenter study of antimicrobial resistance and typing of hospital Staphylococcus aureus isolates from 21 laboratories in 19 countries or states. Microb Drug Resist. 2004;10:160-8.

20 European Committee on Antimicrobial susceptibility testing. Breakpoint tables for interpretation of MIC and zone diameters. Version 10.0 Valid from. 2020 Jan 1.

21 Harmsen D, Claus H, Witte W, Rothgänger J, Claus H, Turnwald D, et al. Typing of methicillin-resistant Staphylococcus aureus in a university hospital setting by using novel software for spa repeat determination and database management. J Clin Microbiol. 2003;41:5442-8.

22 Monecke S, Jatzwauk L, Weber S, Slickers P, Ehricht R. DNA microarray-based genotyping of methicillin-resistant Staphylococcus aureus strains from Eastern Saxony. Clin Microbiol Infect. 2008;14:534-45. 
23 Castanheira M, Watters AA, Mendes RE, Farrell DJ, Jones RN. Occurrence and molecular characterization of fusidic acid resistance mechanisms among Staphylococcus spp. from European countries (2008). J Antimicrob Chemother. 2010;65:1353-8.

24 Edslev SM, Clausen ML, Agner T, Stegger M, Andersen PS. Genomic analysis reveals different mechanisms of fusidic acid resistance in Staphylococcus aureus from Danish atopic dermatitis patients. J Antimicrob Agents Chemother. 2011;55:1173-6.

25 Chen HJ, Hung WC, Tseng SP, Tsai JC, Hsueh PR, Teng LJ. Fusidic acid resistance determinants in Staphylococcus aureus clinical isolates. Antimicrob agents Chemother. 2010; 54:4985.

26 Aldasouqi R, Abu-Qatouseh LF, Badran EF, Alhaj Mahmoud SA, Darwish RM. Genetic determinants of resistance to fusidic acid among Staphylococcus aureus isolates in Jordan. Jundishapur J Microbiol. 2019;12(3): e86120.

27 Yu F, Liu Y, Lu C, Lv J, Qi X, Ding Y, et al. Dissemination of fusidic acid resistance among Staphylococcus aureus clinical isolates. BMC Microbiol. 2015;15:210.

28 Chen CM, Huang M, Chen HF, Ke SC, Li $\mathrm{CR}$, Wang $\mathrm{JH}$, et al. Fusidic acid resistance among clinical isolates of methicillin-resis- tant Staphylococcus aureus in a Taiwanese hospital. BMC Microbiol. 2011 May 12;11: 98.

29 Abouelfetouh A, Kassem M, Naguib M, ElNakeeb M. Investigation and treatment of fusidic acid resistance among methicillin-resistant staphylococcal isolates from Egypt. Microb Drug Resist. 2017;23:8-17.

30 Katopodis GD, Grivea IN, Tsantsaridou AJ, Pournaras S, Petinaki E, Syrogiannopoulos GA. Fusidic acid and clindamycin resistance in community-associated, methicillin-resistant Staphylococcus aureus infections in children of central Greece. BMC Infect Dis. 2010 Dec 13;10:351. 